\title{
Geometry and Inverse Optimality in Global Attitude Stabilization
}

\author{
S. Bharadwaj *, M. Osipchuk ${ }^{\dagger}$, K. D. Mease $\stackrel{+}{+}$, F. C. Park \\ Department of Mechanical and Aerospace Engineering \\ University of California, Irvine, CA 92697-3975
}

\begin{abstract}
The problem of globally stabilizing the attitude of a rigid body is considered. Topological and geometric properties of the space of rotations relevant to the stabilization problem, are discussed. Chevalley's exponential coordinates for a Lie group are used to represent points in this space. An appropriate attitude error is formulated and used for control design. A control Lyapunov function approach is used to design globally stabilizing feedback laws that have desirable optimality properties. Their performance is compared to the performance of previously developed proportional-derivative type control laws. The new control laws achieve the same or greater stabilization rate with less control effort. Special issues in the Lyapunov stability proofs due to the topology of the space of rotations are identified and resolved. The simpler problem of stabilization

${ }^{*}$ Graduate Student; Student Member AIAA

${ }^{\dagger}$ Postdoctoral Researcher

${ }^{\ddagger}$ Associate Professor; Associate Fellow of AIAA

${ }^{\S}$ Copyright (C) by the authors, Published by AIAA with permission

I Assistant Professor; currently, Assistant Professor, Seoul National University, S. Korea
\end{abstract}


on the space of planar rotations is treated throughout the paper to provide insight.

\section{Introduction}

The problem of controlling the orientation of a rigid body is central to the control of aircraft and spacecraft. Large angle maneuvers have long been of interest for satellite control and have more recently become relevant for agile aircraft and missiles. In this paper, we address the attitude control of a rigid body subject only to torques due to its own inertia and to control effectors, either thrusters or momentum wheels, but much of the discussion and results are extendable to a rigid body subject to gravitational and aerodynamic torques in addition. We assume the controls can be continuously varied; the results are thus applicable to control by proportional thrusters, momentum wheels, or on-off thrusters operated in a pulse-width, pulse-frequency modulation mode. The particular control problem we consider is the global feedback stabilization of a specified inertial pointing direction. The stabilization problem provides a sufficiently rich context for the points we wish to make, though our discussion and results could be extended to the global asymptotic tracking problem.

There are two features of the global attitude stabilization problem that motivate our study. First, the state space is a manifold that is not equivalent to a linear vector space. The dynamics of the orientation of a rigid body evolve on the tangent bundle to the rotation group $S O(3)$. $S O(3)$ is a compact manifold without boundary. This topology has profound implications for control design. We approach the formulation and solution of the global stabilization problem from a geometric perspective of $S O(3)$ as both a Riemannian manifold and a Lie group. Chevalley's canonical coordinates for a Lie group are used to represent points in $S O(3)$. The coordinates themselves are not new; they are the elements of what is usually called the principal rotation 
vector. ${ }^{1}$ The geometric perspective is prominent in the approaches to attitude control by Meyer, ${ }^{2}$ Crouch, ${ }^{3}$ Koditschek, ${ }^{5}$ Wen and Kreutz-Delgado, ${ }^{6}$ Paielli and Bach ${ }^{7}$ and Bullo and Murray. ${ }^{8}$ In this paper, we bring together results from these references to clarify the issues in global attitude stabilization and set the stage for our development of new globally stabilizing control laws.

The second feature of the global attitude stabilization problem that motivates our study is the nonlinearity of the attitude dynamics. Two types of feedback laws have been shown to achieve global (or almost global) stabilization. The first is a proportional-derivative law with an extra term that cancels the nonlinear gyroscopic torque..$^{7,9,10}$ This control law is derived using feedback linearization (or a restricted form of it called dynamic inversion or computed torque) and is thus referred to as the feedback linearizing (FBL) control law. The resulting closed-loop dynamics are either partially or completely linearized, in the absence of control saturation and modeling error. The second feedback ${ }^{6,11,12}$ is a linear PD law; the gyroscopic torque is not canceled. From an energy perspective, it is clear that the gyroscopic torque is a neutral party in the stabilization task and in this regard does not need to be canceled.

From a performance point of view, neither of these stabilizing control laws may be satisfactory. The performance of the FBL control law is relatively easy to predict and adjust by tuning the gains, since the closed-loop system is linear or at least partially linear. But global linear performance may not be desirable performance, especially at the expense of canceling the gyroscopic torque. The PD control law does not incur the penalty for canceling the gyroscopic torque, but tuning the PD gains is not straightforward since the closed-loop error dynamics are nonlinear; moreover, it may not be possible to find constant gains that yield uniformly desirable performance. For both laws, large attitude or angular velocity errors require large control effort due to the linear nature of the control laws. Without using some form of gain scheduling, the 
linear structure prohibits scaling back the control response for large errors without compromising the response for small errors.

The obvious means of obtaining a stabilizing control law with good performance is to use a control law that optimizes an appropriate performance measure. For specific initial attitude and angular velocity errors, one can solve a two-point boundary value problem for the optimal control law. Many numerical solutions for minimum time and minimum control energy performance can be found in the literature. ${ }^{13,14}$ To obtain an optimal feedback law, one must essentially solve a Hamilton-JacobiBellman (HJB) partial differential equation. Computing optimal controls is generally considered infeasible for operational attitude control systems. Eigenaxis maneuvers, which are near optimal with respect to time and/or control effort in certain cases, have been used. ${ }^{15}$ Vadali $^{16}$ and Tsiotras ${ }^{17}$ have exploited the cascade structure of the attitude kinematics and dynamics to obtain feedback laws that have certain optimality properties.

A new method ${ }^{18,19}$ offers a feasible approach to obtaining an optimal feedback law. It will be referred to here as the method of inverse optimality. It is wellknown that, for an optimal stabilization problem, the solution to the appropriate HJB equation can serve as a Lyapunov function for proving global stability. In the inverse optimality method, one begins with a control Lyapunov function, uses it to determine a stabilizing feedback control; the theory behind the method shows that the Lyapunov function is the solution to the HJB equation corresponding to an appropriate cost function. For attitude stabilization, an appropriate cost function should include a penalty for attitude and angular velocity errors and a penalty for control effort.

In this paper, new globally stabilizing attitude control laws are derived using the inverse optimality method. The performance of these laws is compared to the performance of existing PD and FBL laws. Our proofs of global stability for the various control laws are based on Lyapunov's direct method. Special considerations are re- 
quired to account for the topology of $S O(3)$. The simpler case of planar rotations, for which the configuration space is $S O(2)$, is used to develop insight throughout the paper. We have presented aspects of this research in conference papers. ${ }^{20,21}$ Other variations of the application of the inverse optimality method to global attitude stabilization have also appeared in the literature. ${ }^{22}$

\section{Rigid Body Rotation: Coordinates, Kinematics and Dynamics}

The configuration space for rigid body rotation is the special orthogonal rotation group, referred to as $S O(3)$ and defined by $S O(3)=\left\{R \in \mathbb{R}^{3 \times 3}: R^{T} R=I, \operatorname{det}(R)=\right.$ $1\}$. Each point in the space is a rotation operator $R$. $R$ describes the relative orientation of a principal axis body frame and an inertial frame. Our convention is that $R$ rotates the body frame into alignment with the inertial frame. Once the two frames are chosen, $R$ can be given in explicit form as the $3 \times 3$ direction-cosine matrix. A single set of global coordinates does not exist. Including coordinate switching as part of a control law would be undesirable; using a single set of coordinates that is almost global is a better alternative.

One popular set of almost global coordinates is the unit quaternion. The unit quaternion is composed of four coordinates; but, since they are linked by a constraint, there are only three independent coordinates. The quaternions actually represent points in the special unitary group $S U(2)$, which can be identified with $S^{3}$, the surface of a unit 3-sphere embedded in $\mathbb{R}^{4}$. Only half of this sphere is needed for a one-to-one correspondence with points in $S O(3)$, thus the mapping from quaternions to $S O(3)$ is two-to-one. This feature manifests itself in the well-known sign ambiguity: for any quaternion representation of a point in $S O(3)$, one can switch the sign of each of the four coordinates and obtain an alternate representation of the same point. Resolving the sign ambiguity is a necessary aspect of using quaternions. Nonetheless, they are 
often used and have attributes that make them particularly well-suited for attitude estimation. ${ }^{1}$

For the design of attitude control laws, it may be advantageous to use a three parameter representation of $S O(3)$, i.e., a minimal parameterization. When control on all of $S O(3)$ is required, one would like coordinates that fail to be global coordinates in as convenient a manner as possible. Of the many ${ }^{1}$ three-coordinate representations for $S O(3)$, the modified Rodriguez parameters and the principal rotation vector seem especially well-suited. The key attribute, both of these coordinate representations share, is that the Jacobian matrix that appears in the corresponding attitude kinematic equations is globally nonsingular. The same cannot be said for the Euler angles, for example. Modified Rodriguez parameters have been used for attitude control design by Tsiotras $^{17}$ and Schaub et. al. ${ }^{15}$ In this paper, we use the principal rotation vector, a special case of Chevalley's canonical coordinates (of the first kind) for a Lie group, in this case the Lie group being $S O(3)$.

Chevalley's canonical coordinates ${ }^{23}$ for a matrix Lie group arise from the exponential map and its inverse, the $\log (\cdot)$ map. For $S O(3)$, these take the form

$$
\begin{gathered}
R=\exp [r]=I+\frac{\sin \|r\|}{\|r\|}[r]+\frac{1-\cos \|r\|}{\|r\|^{2}}[r]^{2} \\
{[r]=\log (R)=\frac{\phi}{2 \sin \phi}\left(R-R^{T}\right)}
\end{gathered}
$$

where $\|\cdot\|$ is the standard Euclidean norm, $\phi$ is defined by $1+2 \cos \phi=\operatorname{Trace}(R)$, and $r \in \mathbb{R}^{3}$. We use $[r]$ to denote the skew-symmetric matrix

$$
[r]=\left[\begin{array}{ccc}
0 & -r_{3} & r_{2} \\
r_{3} & 0 & -r_{1} \\
-r_{2} & r_{1} & 0
\end{array}\right]
$$

such that $[r] v=r \times v$ for all $v \in \mathbb{R}^{3}$. Let $B=\left\{r \in \mathbb{R}^{3}:\|r\| \leq \pi\right\}$, i.e., $B$ is the solid ball of radius $\pi$ centered at the origin in $\mathbb{R}^{3}$. If $r$ is limited to values in $B$, then for 
every point in $S O(3)$ there is a corresponding point in $B$, i.e., the exponential map is onto. Except for the surface of $B$, the exponential map is also one-to-one. On the surface of $B$, the exponential map is two-to-one.

We can write $r=\phi \hat{e}$ where $\hat{e}$ is the unit eigenaxis, i.e., the unit eigenvector of $R$ corresponding to the unity eigenvalue, and $\phi$ is the rotation angle, $-\pi \leq \phi \leq \pi$, from the identity to $R$ about the eigenaxis. This the usual interpretation of $r$ as the principal rotation vector. For each eigenaxis direction, rotations of $\pi$ and $-\pi$ radians lead to the same orientation $R$, but these rotations correspond to distinct, diametrically opposed points on the surface of $B$. Consequently, the log map is double-valued, $\log (R)= \pm \pi[\hat{e}]$, on the surface of $B$. Points on the surface of $B$ satisfy the condition Trace $(R)=-1$. Henceforth we shall refer to the components of $r$ as the exponential coordinates.

The rotational dynamics for a rigid body with fully actuated controls are given by

$$
\begin{array}{rlc}
\dot{R} & =\quad R[\omega] \\
M \dot{\omega} & =[\omega] M \omega+\tau
\end{array}
$$

where $M$ is the inertia matrix, $\tau$ is the control torque, $\omega$ is the angular velocity in body axis coordinates and $[\omega]$ is the corresponding skew-symmetric matrix. The state space for rigid body rotations is the six-dimensional product space of rotation operators and rotation rates $S O(3) \times \mathbb{R}^{3}$. The components of the angular velocity vector in body axes, $\omega$, are the usual coordinates for $\dot{R}$. Instead, we use the state vector expressed as $(r, \dot{r}) \in B \times \mathbb{R}^{3}$.

To express the kinematic equations in the exponential coordinates we require the Jacobian $J(r)$ that relates the time derivatives of the exponential coordinates $\dot{r}$ to the angular velocity $\omega$, as $\omega=J(r) \dot{r}$. This Jacobian is obtained by differentiating the 
exponential map. The Jacobian and its inverse are given by ${ }^{24}$

$$
\begin{aligned}
J(r) & =I+\frac{1-\cos \|r\|}{\|r\|^{2}}[r]+\frac{\|r\|-\sin \|r\|}{\|r\|^{3}}[r]^{2} \\
J^{-1}(r) & =I-\frac{1}{2}[r]+\left(\frac{1}{\|r\|^{2}}-\frac{1+\cos \|r\|}{2\|r\| \sin \|r\|}\right)[r]^{2}
\end{aligned}
$$

The rotational dynamics, Eq. (3) in the state variables $(r, \omega)$ are given by

$$
\begin{aligned}
\dot{r} & =J^{-1}(r) \omega \\
M \dot{\omega} & =[\omega] M \omega+\tau
\end{aligned}
$$

The dynamics can also be expressed in Lagrangian form. ${ }^{11}$ This form can be derived either by starting with the rotational kinetic energy expressed in $r$ and $\dot{r}$ and following the procedure of Lagrangian dynamics or by the following simpler approach. ${ }^{11}$ Differentiating $J(r) \dot{r}=\omega$ with respect to time, pre-multiplying the resulting equation by $J^{T}(r) M$ and using the property $[w] v=-[v] w$, we obtain the second-order form of the attitude dynamics

$$
H(r) \ddot{r}+C(r, \dot{r}) \dot{r}=u
$$

where $H(r)=J^{T} M J, u=J^{T} \tau, C(r, \dot{r})=J^{T} M \dot{J}+J^{T}[M J \dot{r}] J$ and

$$
\begin{aligned}
\dot{J} & =-\frac{r^{T} \dot{r}}{\|r\|^{4}}(2 \cos \|r\|+\|r\| \sin \|r\|-2)[r]+\frac{\|r\|-\sin \|r\|}{\|r\|^{3}}([\dot{r}][r]+[r][\dot{r}]) \\
& +\frac{r^{T} \dot{r}}{\|r\|^{5}}(3 \sin \|r\|-\|r\| \cos \|r\|-2\|r\|)[r]^{2}
\end{aligned}
$$

To complete the description of the state $(r, \dot{r})$ evolution on $B \times \mathbb{R}^{3}$, we specify instantaneous jump conditions which apply when the rigid body either begins with a configuration on the surface of the $\pi$-ball $B$, or reaches it, with an angular velocity that has a positive component along the radial direction $r$. The jump conditions, using superscripts - and + to refer to values before and after the jump, are given by

$$
\begin{aligned}
& r^{+}(t)=-r^{-}(t) \\
& \dot{r}^{+}(t)=\dot{r}^{-}(t)
\end{aligned}
$$


and are applied when $\|r\|=\pi$ and $\dot{r}^{T} r>0$, with no time elapsing during the jump. For example, the jump conditions dictate that a trajectory $r(t)$ that reaches the surface of $B$ with a transverse intersection will jump to the diametrically opposed point and proceed back into the ball from that side. In short, the state evolution is determined by the rule : if $\|r\|=\pi$ and $\dot{r}^{T} r>0$ then apply the jump conditions, Eq.(8), otherwise apply the differential equations, Eq.(6). Note that $\dot{r}^{T} r$ will not be positive after the jump conditions are applied since $\dot{r}^{+^{T}} r^{+}=-\dot{r}^{-T} r^{-}$. The subsequent state evolution after the application of the jump conditions is therefore always dictated by the differential equations; the jump conditions will never cause "chattering" between two diametrically opposed points on the surface of $B$. Note also that since the Jacobian $J(r)$ is nonsingular even on the surface of the $\pi$-ball $B$, the kinematic equation $\dot{r}=J^{-1}(r) \omega$ is well defined everywhere on $B$ including its surface.

\section{Global Stabilization Problem}

The attitude stabilization problem considered here is to determine a feedback control law that renders a desired constant inertial pointing direction a globally asymptotically stable equilibrium point. We assume that the inertial frame referred to in defining the rotation matrix $R$ is such that when the body frame is in alignment with the inertial frame, as indicated by $R=I$, the desired pointing direction is achieved. Thus, the stabilization problem is to determine a feedback control law that renders the point $(R, \omega)=(I, 0)$ a globally asymptotically stable equilibrium. In terms of the exponential coordinates, the equilibrium point is given by $(r, \dot{r})=(0,0)$.

Bhatia $^{25}$ has shown that for an n-dimensional dynamical system with a continuous (or smoother) control law, the domain of attraction of an asymptotically stable equilibrium point is globally homeomorphic to $\mathbb{R}^{n}$. Since the state space $S O(3) \times \mathbb{R}^{3}$ is not globally homeomorphic to $\mathbb{R}^{6}$, it follows that topological restrictions preclude 
global asymptotic stabilization by continuous feedback. Koditschek ${ }^{5}$ has shown that the system, Eqs. (5) or (6), under any continuous feedback law that asymptotically stabilizes the equilibrium point of interest, has a minimum of three other spurious equilibria. There exist continuous control laws such that this minimum of three spurious equilibria is achieved, and such that the spurious equilibria are unstable. Thus continuous control laws exist that drive all initial states, except for a set of measure zero, to the desired rest equilibrium attitude.

A useful example for understanding these results is stabilization on $S O(2)$, the space of planar rotations. The points of $S O(2)$ can be identified with the points on a unit circle, i.e., $S^{1}$. We use $\theta \in[-\pi, \pi]$ as the configuration coordinate; $\theta=\pi$ and $\theta=-\pi$ correspond to the same point. Hence, $\theta$ fails as a global coordinate for $S O(2)$, similar to how $r$ fails for $S O(3)$. For the simple first-order system $\dot{\theta}=u$, the control law $u=-\sin \theta$ is continuous with an asymptotically stable equilibrium at $\theta=0$ and an unstable equilibrium at $\theta=\pi$ (and $-\pi$ ). The domain of attraction for $\theta=0$ is the whole circle except the unstable equilibrium point. Any continuous control law will have at least one such spurious equilibrium point. On the other hand, there exist discontinuous control laws that globally stabilize the point $\theta=0$. An example is $u=-\theta$. The discontinuity is at the point $\theta=\pi($ and $-\pi)$.

Consider the stabilization of a second-order system $\ddot{\theta}=u$ on $S O(2)$ by the PD law $u=-k_{p} \theta-k_{d} \dot{\theta}$ with positive constant gains. The jump conditions required to complete the description of the state evolution are given by $\theta^{+}=-\theta^{-}$and $\dot{\theta}^{+}=\dot{\theta}^{-}$ and are applied when $|\theta|=\pi$ and $\theta \dot{\theta}>0$. Global stability can be proved using the Lyapunov function

$$
V=\frac{1}{2} \dot{\theta}^{2}+\gamma \theta \dot{\theta}+\frac{1}{2}\left(k_{p}+\gamma k_{d}\right) \theta^{2}
$$

The derivative of $V$ along a trajectory of the closed-loop system is

$$
\dot{V}=\left(-k_{d}+\gamma\right) \dot{\theta}^{2}-\gamma k_{p} \theta^{2}
$$


except at a jump. Note that, with $\gamma=0, \dot{V}$ is independent of $\theta$ and one must appeal to LaSalle's invariance principle to show stability. The cross-term ${ }^{6}$ avoids this and also allows an exponential bound on the stabilization rate. By choosing $\gamma$ positive but less than $k_{d}, V$ can be made positive definite and $\dot{V}$ can be made negative definite everywhere on $[-\pi, \pi] \times \mathbb{R}$. (The negative definiteness of $\dot{V}$ is also required for $V$ to serve as a control Lyapunov function, as will be discussed later.) From the jump conditions, it follows that $V^{+}<V^{-}$, where $V^{+}=V\left(\theta^{+}, \dot{\theta}^{+}\right)$and $V^{-}=V\left(\theta^{-}, \dot{\theta}^{-}\right)$. Thus $V$ decreases along all trajectories of the closed-loop system, and we can conclude that the point $(\theta, \dot{\theta})=(0,0)$ is globally asymptotically stable.

Consider instead the representation of $S O(2)$ by the coordinates $(x, y) \in \mathbb{R}^{2}$ subject to the constraint $\sqrt{x^{2}+y^{2}}=1$. If the possible values for $(x, y)$ are generated by $(x, y)=(\cos (\theta / 2), \sin (\theta / 2))$ with $\theta$ ranging from $-2 \pi$ to $2 \pi$, the $(x, y)$ coordinates for $S O(2)$ are analogous to the quaternion coordinates for $S O(3)$. The map from $(x, y)$ to $S O(2)$ is two to one. Applying the proportional feedback $u=-k_{p} y=-k_{p} \sin (\theta / 2)$ to the first-order system $\dot{\theta}=u$ with $\theta \in[-2 \pi, 2 \pi]$, we see that there are equilibria at $(1,0)$ and $(-1,0)$ and that $u$ is continuous. The equilibrium $(1,0)$ is stable, whereas the equilibrium $(-1,0)$ is unstable. Both $(1,0)$ and $(-1,0)$ represent the same point in $S O(2)$. For an initial condition very near $(-1,0)$, the control law will take the configuration through an almost $360^{\circ}$ rotation to reach $(1,0)$. To avoid this, one has to either flip the sign on the feedback so that $(-1,0)$ is the asymptotically stable equilibrium or choose the physically equivalent initial condition with the opposite sign. For example, if the value of $(x, y)$ is always chosen such that $x>0$, then the resulting control law on $S O(2)$ is discontinuous. A discontinuity would also be introduced if a rule for changing the sign of the gain were used. Thus the continuous control law with two equilibria in the $(x, y)$-space is mapped to a globally asymptotically stable, but discontinuous, control law on $S O(2)$.

The situation analogous to the one described in the previous paragraph arises 
with quaternions. Wen and Kreutz-Delgado ${ }^{6}$ develop a continuous control law on $S^{3} \times \mathbb{R}^{3}$, where $S^{3}$ (or equivalently $S U(2)$ ) is the space corresponding to quaternions, that yields two equilibria : one asymptotically stable, the other an unstable repellor. When mapped onto $S O(3) \times \mathbb{R}^{3}$, using appropriate logic for either choosing the sign of the quaternion or the sign of the proportional feedback gain, the resulting control law is globally asymptotically stable and discontinuous.

Using exponential coordinates, we formulate feedback laws on $B \times \mathbb{R}^{3}$. The mapping between $B$ and $S O(3)$ is one-to-one in the interior of $B$, but requires an arbitrary choice between two representations on the boundary $\partial B$ where the attitude is 180 degrees away from the desired attitude about some axis. However, the two representations lead to the same control action and the same physical trajectory, in contrast to the situation for the quaternion based control laws. The feedback control laws in exponential coordinates have discontinuities corresponding to the jumps in $r$. The Lyapunov function approach will be used to prove the global asymptotic stability of the discontinuous control laws in later sections of the paper. The Lyapunov functions we use are discontinuous. Similar to the $S O(2)$ example, we will show that the Lyapunov function decreases along all trajectories of the closed-loop system, including across jumps.

\section{Attitude Error Definition}

The configuration space of planar rotations, $S O(2)$, is a one-dimensional compact manifold without boundary. From a given initial position there are two paths back to the desired position, a situation quite different from the case where the configuration space is the one-dimensional real line. The shortest path can be defined as the minimum angle path and is easily determined. To develop feedback laws on the $S O(3)$ configuration space, it is desirable to know the shortest path between two attitudes. 
Which path is the shortest depends on how path lengths are measured, i.e., on the definition of a metric on $S O(3)$.

A Riemannian metric for $S O(3)$ is established by a smooth assignment of an inner product to the tangent space at each point. Using the metric defined by $\langle\dot{R}, \dot{R}\rangle_{R} \triangleq$ $\frac{1}{2} \operatorname{trace}[\omega]^{T}[\omega]=\omega^{T} \omega$, the length of a differentiable curve $R(t), 0 \leq t \leq t_{f}$, on $S O(3)$ is given by

$$
L=\int_{0}^{t_{f}}\langle\dot{R}(t), \dot{R}(t)\rangle_{R(t)}^{\frac{1}{2}} d t=\int_{0}^{t_{f}}\left(\omega^{T} \omega\right)^{1 / 2} d t
$$

Between any two points on $S O(3)$, the shortest path is the path connecting the two points that minimizes $L$. It is often easier to write the Euler-Lagrange equations for the functional

$$
E=\int_{0}^{1}\langle\dot{R}(s), \dot{R}(s)\rangle_{R(s)} d s=\int_{0}^{1} \omega^{T} \omega d s
$$

For any two points in $S O(3)$, i.e., for any two attitudes, it can be shown that the shortest path connecting them also minimizes $E$. The path minimizing $E$ is called the minimal geodesic, and its parameter $s$ will be affinely related to arclength. ${ }^{26}$

The geodesics for the above metric are easily described using the exponential coordinates. The minimal geodesic from point $I$ to point $R_{1}=\exp \left\{\left[r_{1}\right]\right\}$ on $S O(3)$ is given by $R(s)=\exp \left\{\left[r_{1}\right] s\right\}$ for $0 \leq s \leq 1$. This is an eigenaxis rotation: $\phi=\left\|r_{1}\right\|$ is the eigenaxis rotation angle and $r_{1} /\left\|r_{1}\right\|$ is the unit vector in the direction of the eigenaxis expressed in body axis coordinates. From the viewpoint of $r$ representing points in a ball of radius $\pi$ in $\mathbb{R}^{3}$, segments of radial lines emanating from the origin in the ball map into (using the exponential map) the geodesics emanating from the identity element of $S O(3)$. We note that the geodesic from an arbitrary attitude $R_{0}$ to an arbitrary attitude $R_{2}$ is the left translation by $R_{0}$ of the geodesic from $I$ to $R_{0}^{T} R_{2}$, i.e., the geodesic is given by $R(s)=R_{0} \exp \{[r] s\}$, where $[r]=\log \left(R_{0}^{T} R_{2}\right)$ and $R(1)=R_{2}$. This result is needed to formulate the tracking problem and follows from 
the left-invariance of the metric. ${ }^{26}$ Thus the exponential coordinates provide all the important information regarding the minimal geodesics for the particular metric we have defined. Other metrics could be used, but the corresponding minimal geodesics will in general be more complicated to determine and it does not seem that there would be any benefit.

Consider first the stabilization of a mechanical system with configuration space $\mathbb{R}^{n}$. A first-order system of the simple form $\dot{x}=u$ is stabilized at the origin by proportional error feedback of the form $u=-K_{p} x$ with positive definite gain matrix $K_{p}$. A second-order system of the simple form $\ddot{x}=u$ is stabilized by PD error feedback of the form $u=-K_{p} x-K_{d} \dot{x}$ with both gain matrices positive definite. The proportional feedback term is constructed from the position error $e=x-0$, which is just $x$ since we are stabilizing the origin. The interpretation of $x$ that seems most appropriate in generalizing proportional control to $S O(3)$ is that $x$ is the vector that is tangent to the minimum geodesic path from the current configuration to the desired configuration and whose magnitude is equal to the length of this path. ${ }^{27}$ For the configuration space $S O(3)$, the corresponding tangent vector would have direction along the minimal geodesic connecting the current attitude $R$ to the desired attitude $I$ and magnitude equal to the length of this minimal error geodesic path.

In the following sections, we design several stabilizing control laws. In doing so, we use $r$ to represent the attitude error. For a second-order system on $S O(3)$ our PD law has the form $u=-K_{p} r-K_{d} \dot{r}$. The vector $r$ is tangent to the geodesic leading to the desired orientation on $S O(3)$, and its magnitude is equal to the length of this path. Thus, the feedback term $K_{p} r$ is the natural generalization to $S O(3)$ of proportional feedback on $\mathbb{R}^{n}$. Given that $r=\phi \hat{e}$ and that the unit quaternion $q=(\cos (\phi / 2), \sin (\phi / 2) \hat{e})$, the proportional feedback term $K_{p} \sin (\phi / 2) \hat{e}$, using the vector part of the quaternion, is similar and has been used in other studies. ${ }^{6,10}$ To develop a cost function for optimal attitude maneuvers, Schaub et. a ${ }^{28}$ address the 
issue of attitude error formulation and resolve it by a different but related approach.

\section{PD Control of Almost Feedback Linearized System}

Feedback linearization has been used to derive globally stabilizing control laws with regard to the first-order form of Eq.(5). The angular velocity equations can be linearized by a control transformation. ${ }^{6,7,10}$ The combined angular velocity and kinematic equations can be linearized by state and control transformations; results are available for attitude representations in Euler angles ${ }^{29}$ the vector part of the quaternion ${ }^{7,9}$ and exponential coordinates. ${ }^{20}$ Strictly speaking ${ }^{5}$ the combined system can not be feedback linearized globally, because global coordinates do not exist.

The feedback linearization of the second-order form, Eq. (6), requires only the control transformation

$$
u=H(r) v+C(r, \dot{r}) \dot{r}
$$

In terms of the new control variable $w$, the dynamics take the form of three scalar, decoupled, double integrator systems. In vector form, we have $\ddot{r}=v$. The jump conditions, Eq.(8), are required to complete the description of the state evolution. A control law of the form $w=-K_{p} r-K_{d} \dot{r}$ with $K_{p}$ and $K_{d}$ positive definite matrices, globally asymptotically stabilizes the state $(r, \dot{r})=(0,0)$. Applying the transformation Eq. (11), we obtain the control law

$$
u_{F B L}=-H(r) K_{p} r-H(r) K_{d} \dot{r}+C(r, \dot{r}) \dot{r}
$$

Global stability is proved using the Lyapunov function

$$
V_{F}=\frac{1}{2} \dot{r}^{T} \dot{r}+\frac{1}{2} r^{T}\left(K_{p}+\gamma K_{d}\right) r+\gamma \dot{r}^{T} r
$$

where $0<\gamma<\min \left\{\sqrt{\lambda_{\min }\left(K_{p}\right)}, \lambda_{\min }\left(K_{d}\right)\right\}$. It can be shown that on $B \times \mathbb{R}^{3}, V_{F}$ is positive definite on $B \times \mathbb{R}^{3}$ and excluding jump points, $\dot{V}_{F}$ is negative definite along 
all trajectories of the closed-loop system. From the jump conditions, Eq.(8), it follows that $V_{F}^{+}<V_{F}^{-}$across any jump between diametrically opposed points on the surface of the $\pi$-ball. Therefore, $V_{F}$ is strictly decreasing along every trajectory of the closed loop system, and we conclude that the origin of the closed-loop system is globally asymptotically stable.

\section{PD Control of the Nonlinear System}

The PD control law is

$$
u_{P D}=-K_{p} r-K_{d} \dot{r}
$$

with $K_{p}$ and $K_{d}$ symmetric, positive definite gain matrices. To prove that this control law globally asymptotically stabilizes the system Eq.(6), we use the Lyapunov function with a cross-term ${ }^{6}$

$$
V_{E}=\frac{1}{2} \dot{r}^{T} H(r) \dot{r}+\frac{1}{2} r^{T}\left(K_{p}+c K_{d}\right) r+c r^{T} H(r) \dot{r}
$$

with $0<c<\min \{\sqrt{\mu}, \nu\}$ where $\mu=\inf _{r \in B} \lambda_{\min }\left(H^{-1}(r) K_{p}\right)$ and

$$
\nu=\lambda_{\min }\left(K_{d}\right) /\left(\sup _{r \in B} \lambda_{\max }(H(r))+k \pi\right)
$$

where $k$ is defined below. It can be shown that $V_{E}$ is positive definite for the above choice of $c$ by rewriting it as

$$
V_{E}=\frac{1}{2}\left(\begin{array}{ll}
r^{T} & \dot{r}^{T}
\end{array}\right) P_{c}\left(\begin{array}{l}
r \\
\dot{r}
\end{array}\right), \text { where } P_{c}=\left(\begin{array}{cc}
K_{p}+c K_{d} & c H(r) \\
c H(r) & H(r)
\end{array}\right)
$$

and using the Schur complement formula along with the fact that $H(r)$ is symmetric and positive definite. The time derivative of $V_{E}$ along a solution of the system Eq.(6), under the PD control law is

$$
\dot{V}_{E}=-\dot{r}^{T} K_{d} \dot{r}+c \dot{r}^{T} H(r) \dot{r}-c r^{T} K_{p} r+c r^{T}(\dot{H}(r)-C(r, \dot{r})) \dot{r}
$$


Using $\dot{H}(r)-C(r, \dot{r})=C(r, \dot{r})^{T}$, we get

$$
\dot{V}_{E}=-\dot{r}^{T} K_{d} \dot{r}+c \dot{r}^{T} H(r) \dot{r}-c r^{T} K_{p} r+c \dot{r}^{T} C(r, \dot{r}) r \triangleq-\sigma_{E}
$$

Each of the above terms can be bounded as shown : $-\dot{r}^{T} K_{d} \dot{r} \leq-\lambda_{\min }\left(K_{d}\right)\|\dot{r}\|^{2}$, $\dot{r}^{T} H(r) \dot{r} \leq\left(\sup _{r \in B} \lambda_{\max }(H(r))\right)\|\dot{r}\|^{2},-r^{T} K_{p} r \leq-\lambda_{\min }\left(K_{p}\right)\|r\|^{2} \leq-\lambda_{\min }\left(K_{p}\right) \pi^{2}$ and $\dot{r}^{T} C(r, \dot{r}) r \leq\|C(r, \dot{r})\|\|r\|\|\dot{r}\|$. It is important to note that the Coriolis term $C(r, \dot{r})$ can be bounded ${ }^{30}$ by $\|C(r, \dot{r})\| \leq k\|\dot{r}\|$. This can be justified using the expressions in Eq.(7). Hence $\|C(r, \dot{r})\|\|r\| \dot{r}\|\leq k \pi\| \dot{r} \|^{2}$. Therefore,

$$
\dot{V}_{E} \leq-\left(\lambda_{\min }\left(K_{d}\right)-c\left(\sup _{r \in B} \lambda_{\max }(H(r))+k \pi\right)\right)\|\dot{r}\|^{2}
$$

This shows that $\dot{V}_{E}$ is negative definite for the above choice of $c$. From the jump conditions, Eq.(8), it follows that $V_{E}^{+}<V_{E}^{-}$across every jump between diametrically opposed points on the surface of the $\pi$-ball. Therefore $V_{E}$ is strictly decreasing along every trajectory of the closed loop system, and we conclude that the origin of the closed-loop system is globally asymptotically stable.

PD control laws and corresponding stability proofs have appeared in several papers. $6,10,12,31$ We treat the PD law here for comparison to the new control laws developed in the next two sections. There are also some novel aspects to our stability proof. We note that some $e^{6,31}$ of the proofs require that the proportional gain be a scalar. The proportional gain is a matrix gain of a special form in the result of Wie et $a l .{ }^{10}$ The formulation and theory developed by Slotine and Di Benedetto ${ }^{11}$ does not guarantee global asymptotic stability, but rather asymptotic stability within the domain of validity of the Cayley-Rodriguez coordinates. Egeland and Godhavn ${ }^{12}$ prove the global stability of a PD law on $S^{3} \times \mathbb{R}^{3}$ with arbitrary positive definite matrix gains using passivity.

Whereas the Lyapunov function $V_{F}$ contains the term $\frac{1}{2} \dot{r}^{T} \dot{r}$, the Lyapunov function $V_{E}$ contains the kinetic energy $T=\frac{1}{2} \dot{r}^{T} H \dot{r}$. The derivative of $T$ along a trajectory 
of the system in Eq. (6) is $\dot{T}=\frac{1}{2} \dot{r}^{T}[\dot{H}-2 C(r, \dot{r})] \dot{r}+\dot{r}^{T} u$. It can be seen from the expressions Eqs. $(7)$ that $\dot{H}(r)=C(r, \dot{r})+C(r, \dot{r})^{T}$. Consequently, the matrix $\dot{H}-2 C$ is skew symmetric, and hence the first term in $\dot{T}$ is zero. When $u=0$, the kinetic energy is constant. In contrast, using the feedback linearization law of the form in Eq. (11), we have $\dot{T}=\frac{1}{2} \dot{r}^{T} \dot{H} \dot{r}$ when $v=0$. Since $\dot{H}$ is in general not negative semidefinite, canceling the nonlinearity - the gyroscopic torque - can increase $T$. In the context of a particular Lyapunov function, the stabilization task is to determine a feedback control law such that $\dot{V}<0$ for the closed-loop system. This shows that for a Lyapunov function $V$ that includes the kinetic energy, the gyroscopic torque $C(r, \dot{r}) \dot{r}$ has no effect on $\dot{V}$. In this regard, a control law that includes a term to cancel the gyroscopic torque is using control effort that does not contribute to stabilization. (This point has been made in the robotics context ${ }^{4}$ ).

A control law related to Eq. (14) has been developed by Koditschek ${ }^{5}$ from the geometric perspective. He defines a navigation function that gives rise to a gradient vector field on $S O(3)$. He then lifts this vector field to a vector field on the state space $S O(3) \times \mathbb{R}^{3}$, thereby defining a feedback law. The feedback law is continuous and yields almost global stability, meaning that there is only a set of points in the state space of measure zero that are not attracted to the desired rest state. Bullo and Murray $^{27}$ have extended some of Koditschek's results to a more general setting.

\section{Inverse Optimal Stabilizing Control Laws}

PD control laws with and without feedback linearization, while globally asymptotically stabilizing, may have unsatisfactory performance for large initial offsets in attitude and/or angular velocity for the reasons given in the Introduction. We are naturally led to consider control laws that minimize a suitable cost function. The attitude control literature abounds with numerical solutions to optimal control prob- 
lems, predominately minimum time and minimum control effort problems. ${ }^{13}$ It may not be feasible or at least desirable, however, to store or compute optimal solutions for an operational attitude control system.

Eigenaxis maneuvers have been used for a number of operational systems, including those of the Apollo and Shuttle vehicles. The eigenaxis is the optimal rotation axis for the minimum time and minimum control effort rest-to-rest maneuvers in the special case of a spherically symmetric inertia and a spherically symmetric torquing capability, i.e., $\|\tau\| \leq \tau_{\max }$, (in the absence of additional torques as we are assuming in this paper). In the spherically symmetric inertia case, the gyroscopic torque is zero. With $K_{p}=k_{p} H(r)$ and $K_{d}=k_{d} H(r)$, where $k_{p}$ and $k_{d}$ are positive scalars, and starting from rest, the attitude motion under the PD law, Eq.(14), is about the eigenaxis. Wie et al. ${ }^{10}$ have achieved this same effect by constructing the proportional feedback term from the vector part of the unit quaternion.

It has been shown ${ }^{32}$ that with upper and lower bounds on each of three orthogonal torque generators, constraining the control torque vector to lie in a box, the minimumtime maneuver is in general not an eigenaxis rotation, even with a spherically symmetric inertia. A reasonable conjecture is that, in the absence of either spherically symmetric inertia or spherically symmetric torquing capability, or in motion-to-rest maneuvers, the eigenaxis rotation is not in general the minimum-time or minimum control effort maneuver . Eigenaxis rotations may of course be near-optimal in certain cases and serve as good reference maneuvers.

In this section, we take some initial steps in applying the inverse optimality method to obtain globally stabilizing control with good performance. Using the inverse optimality method, we derive control laws that minimize a cost function that includes penalties on state error and control effort. The attitude error is defined as the angular distance about the eigenaxis. Because the cost function also includes penalties on angular velocity and control effort, the optimal maneuvers are not in general eigenaxis 
rotations.

The starting point for the inverse optimality method is the control Lyapunov function $^{19}$ (cLf). For a control system of the form $\dot{x}=f(x)+G(x) u$, with $x \in \mathbb{R}^{n}$ and $u \in \mathbb{R}^{m}, V$ is a cLf, if it is a smooth, positive definite and radially unbounded function of $x$ and if, for $x \neq 0$, it has the property that at any $x$ where the components of $V^{\prime}(x) G(x)$ are all zero, $V^{\prime}(x) f(x)<0$.

If $V$ is a cLf, then the existence of a control that makes $\dot{V}=V^{\prime}(x) f+V^{\prime}(x) G u$ negative, i.e., the existence of a stabilizing control, is assured. For a given continuous, positive definite function $\sigma$ we could compute the control $u(x)$ that achieves a specified stabilization rate $\dot{V}=-\sigma(x)$ whenever $V^{\prime} G \neq 0$. However, for values of $x$ where $V^{\prime}(x) f(x) \leq-\sigma(x)$, it would be better to set $u(x)=0$; otherwise, control effort would be used needlessly to reduce the stabilization rate. Freeman and Kokotovic ${ }^{19}$ proposed that a stabilizing feedback law $u(x)$ can be constructed from the solution at each $x$ to the static quadratic programming problem

$$
\begin{aligned}
\text { minimize } & u^{T} u \\
\text { subject to } & V^{\prime}(x)(f(x)+G(x) u) \leq-\sigma(x)
\end{aligned}
$$

The solution of this problem is

$$
u_{o p t}=-\frac{\lambda}{2}\left[V^{\prime}(x) G(x)\right]^{T}
$$

where

$$
\lambda= \begin{cases}\frac{2\left(V^{\prime}(x) f(x)+\sigma(x)\right)}{V^{\prime}(x) G(x)\left[V^{\prime}(x) G(x)\right]^{T}} & \text { if } V^{\prime}(x) f(x)>-\sigma \\ 0 & \text { if } V^{\prime}(x) f(x) \leq-\sigma\end{cases}
$$

The control $u(x)=u_{\text {opt }}(x)$ is the minimum norm control that achieves the specified stabilization rate or better. It is continuous except possibly at $x=0$, provided that $V^{\prime} f<-\sigma$ whenever all of the components of $V^{\prime} G$ are zero. ${ }^{19}$ 
It has been shown ${ }^{19}$ that the resulting feedback control minimizes a cost function of the form $J(x(0), u)=\int_{0}^{\infty}(Q(x)+W(x, u)) d t$ where the error penalty $Q$ is continuous, positive definite and grows with the magnitude of $x$, and the control penalty $W$ is continuous in $x$ and $u$, and, for each $x$, convex in $u$ with minimum value at $u=0$. The cost function is the nonlinear analogue of the quadratic cost function used in linear quadratic optimal control.

The diagram $J(Q, W) \rightarrow V_{v f} \rightarrow u_{o p t} \leftarrow\left(V_{c L f}, \sigma\right)$ indicates the forward and inverse paths to the optimal control $u_{\text {opt }}$. For conceptual simplicity, assume that $V_{v f}(x)=$ $V_{c L f}(x), \forall x \in \mathbb{R}^{n}$, although this is not the most general case. ${ }^{33}$ The forward path begins with specifying the cost functional via $Q$ and $W$. Then the HJB equation is solved to obtain the value function $V_{v f}$. From $V_{v f}$ the optimal control is obtained. (Alternatively, $u_{\text {opt }}$ could be constructed by obtaining individual optimal trajectories for a range of initial conditions.) The inverse path begins with the specification of the pair $\left(V_{c L f}, \sigma\right)$. The optimal control is computed point-wise by solving the static optimization problem. Solving the static optimization problem is much simpler than solving the HJB equation; however, specifying $\left(V_{c L f}, \sigma\right)$ is generally more difficult than specifying $(Q, W)$. Although we are not addressing robustness in this paper, we note that there is a version of the inverse optimality method that ensures robustness to a class of disturbance inputs. ${ }^{19}$

Our application of the inverse optimality method to global attitude stabilization requires a modification of the cLf definition. We say a Lyapunov function $V(r, \dot{r})$ is a cLf if it is a smooth, positive definite function of $(r, \dot{r}) \in(B-\partial B) \times \mathbb{R}^{3}$, is strictly increasing radially in $B$ for all $\dot{r}$, and is radially unbounded in $\dot{r}$ for all $r \in B$, and if it has the property that at any $(r, \dot{r}) \neq(0,0)$ where the components of $\left(\frac{\partial V}{\partial \dot{r}}\right) H^{-1}$ are all zero, $\left(\frac{\partial V}{\partial r}\right) \dot{r}-\left(\frac{\partial V}{\partial \dot{r}}\right) H^{-1} C \dot{r}<0$ and $V^{+}<V^{-}$for all possible jumps. Note that $\partial B$ denotes the boundary of $B$, i.e., the surface of the $\pi$-ball, and $(B-\partial B)$ refers to the interior of the ball. 


\section{Inverse Optimal Control Law for Planar Rotations}

In order to gain a better understanding of the control laws that the inverse optimal design procedure yields, we design such a control law for stabilizing the second order

system $\ddot{\theta}=u$ on the space of planar rotations $S O(2)$. Since we have shown that the Lyapunov function given by Eq.(9) can be used to prove global stability, it is also a cLf according to the modified definition. In order to compare the resulting inverse optimal control with PD control, we take $\sigma=\left(k_{d}-\gamma\right) \dot{\theta}^{2}+\gamma k_{p} \theta^{2}$, which is consistent with the $\dot{V}$ achieved by a PD control law (see Eq.(10)). In other words, through the inverse optimal design, we seek a controller that achieves the same (or better) stabilization rate as the PD control law. The optimal control law is

$$
u_{o p t}= \begin{cases}-k_{p} \theta-k_{d} \dot{\theta} & \text { if }(\dot{\theta}+\gamma \theta)\left(k_{p} \theta+k_{d} \dot{\theta}\right)>0 \\ 0 & \text { if }(\dot{\theta}+\gamma \theta)\left(k_{p} \theta+k_{d} \dot{\theta}\right) \leq 0\end{cases}
$$

The optimal control law shows that in certain regions of the state space $(\theta, \dot{\theta})$ bounded by the pair of straight lines $\dot{\theta}+\gamma \theta=0$ and $k_{p} \theta+k_{d} \dot{\theta}=0$, the control can be turned off and yet achieve the desired stabilization rate or better. In the complementary regions, the PD control and the optimal control are identical. Fig. 1 shows the state-space trajectories produced by a PD control law and the optimal control law, using identical gains. The optimal trajectory proceeds more directly and quickly to the origin overall, and yet requires less control effort since the control is zero for a significant portion of the trajectory.

In the following two subsections, we show that the Lyapunov functions in Eqs. (13) and (15) are cLf's in our modified sense and use them to design optimal control laws for attitude stabilization on $S O(3)$. For the attitude stabilization problem as formulated in this paper, we use $x=(r, v), v=\dot{r}, f=\left(v,-H^{-1} C\right)$ and $G=\left(0, H^{-1}\right)$ in applying the general result in Eq.(18). 


\section{$V_{F}$ as a Control Lyapunov Function}

The fact that $V_{F}$ could be used to prove the global stability of $u_{F B L}$, Eq. (12), implies that $V_{F}$ is a cLf. The control law $u_{F B L}$ yields the stabilization rate $\sigma_{F}$. Using the result in Eq. (18), the optimal control for the pair $\left(V_{F}, \sigma_{F}\right)$ is $u_{o p t F}=-\lambda_{F} H^{-1}(\dot{r}+\gamma r)$ where

$$
\lambda_{F}= \begin{cases}\frac{(\dot{r}+\gamma r)^{T}\left(K_{p} r+K_{d} \dot{r}-H^{-1}(r) C(r, \dot{r}) \dot{r}\right)}{(\dot{r}+\gamma r)^{T}\left(H^{T}(r) H(r)\right)^{-1}(\dot{r}+\gamma r)} & \text { if } V_{F}^{\prime} f>-\sigma_{F} \\ 0 & \text { if } V_{F}^{\prime} f \leq-\sigma_{F}\end{cases}
$$

By design, $u_{o p t F}$ is the minimum norm control that provides the same stabilization rate $\dot{V}_{F}=-\sigma_{F}$ as the control law $u_{F B L}$ or better.

\section{$V_{E}$ as a Control Lyapunov Function}

The fact that $V_{E}$ could be used to prove the global stability of $u_{P D}$, Eq. (14), implies that $V_{E}$ is a cLf. The control law $u_{P D}$ yields the stabilization rate $\sigma_{E}$. Using the result in Eq. (18), the optimal control for the pair $\left(V_{E}, \sigma_{E}\right)$ is $u_{o p t E}=-\lambda_{E}(\dot{r}+c r)$ where

$$
\lambda_{E}= \begin{cases}\frac{(\dot{r}+c r)^{T}\left(K_{p} r+K_{d} \dot{r}\right)}{(\dot{r}+c r)^{T}(\dot{r}+c r)} & \text { if } V_{E}^{\prime} f>-\sigma_{E} \\ 0 & \text { if } V_{E}^{\prime} f \leq-\sigma_{E}\end{cases}
$$

The three-dimensional control allows freedom for optimization, even when the control is non-zero, in contrast with $S O(2)$ case. As a result, $u_{o p t E}$ is not in general the same as $u_{P D}$ at states where $u_{o p t E} \neq 0$. By design, $\left\|u_{o p t E}\right\| \leq\left\|u_{P D}\right\|$ at these points.

\section{Performance Comparison}

The performance differences between the control laws derived in the preceding sections are illustrated by the following numerical results. As expected, the difference in 
performance between the control law $u_{F B L}$ and the optimal control $u_{\text {optF }}$ is most pronounced when the gyroscopic torque is large. In that case canceling the nondetrimental gyroscopic torque constitutes a substantial part of the control effort. The performances of the two control laws are illustrated in Fig. 2 for the following set of initial conditions and parameters :

$$
M=\left[\begin{array}{ccc}
15 & 5 & 5 \\
5 & 10 & 7 \\
5 & 7 & 20
\end{array}\right] \mathrm{kg} \mathrm{m}^{2}
$$

$r=\phi \hat{e}=2.5 \mathrm{rads}(0.4896,0.2032,0.8480)^{T}, \omega=(1,-1,-1)^{T} \mathrm{rad} / \mathrm{sec} ;$ gains $K_{p}=$ $\operatorname{diag}(1.5,1.5,1.5) \mathrm{kg} \mathrm{m}{ }^{2} / \mathrm{sec}^{2}, K_{d}=\operatorname{diag}(2,2,2) \mathrm{kg} \mathrm{m}^{2} / \mathrm{sec}$, and $\gamma=0.5$. Fig. 2(a) shows that the optimal control $u_{\text {optF }}$ uses less control effort than the feedback linearizing control $u_{F B L}$. Fig. 2(b) shows that the performance of both the control laws in terms of the settling time for $\|r\|$ is similar.

The performance difference between the optimal control $u_{o p t E}$ and the PD control $u_{P D}$ is illustrated in Fig. 3(a) for the following set of initial conditions and parame$\operatorname{ters}^{17}: M=\operatorname{diag}(10,6.3,8.5) \mathrm{kg} \mathrm{m}^{2}, r=\phi \hat{e}=2.5 \operatorname{rads}(0.4896,0.2032,0.8480)^{T}, \omega=$ $(0.1,-0.1,0.1)^{T} \mathrm{rad} / \mathrm{sec} ;$ gains $K_{p}=\operatorname{diag}(2,2,2) \mathrm{kg} \mathrm{m}^{2} / \mathrm{sec}^{2}, K_{d}=\operatorname{diag}(1,1,1) \mathrm{kg} \mathrm{m}^{2} / \mathrm{sec}$, and $c=0.1$. Fig. 3(a) shows that the optimal control $u_{o p t E}$ has much better performance in terms of the settling time for $\|r\|$ than the PD control. Fig. 3(b) shows that the control effort used by $u_{o p t E}$ is much less than that of $u_{P D}$. In fact, $u_{o p t E}$ is mostly zero, indicating that, without any applied control torque, the $\dot{V}$ requirement is being met.

Fig. 4 shows the two trajectories projected onto the state space hyperplane $\{(r, \dot{r})$ : $\left.r_{2}=\dot{r}_{2}=r_{3}=\dot{r}_{3}=0\right\}$. The optimal trajectory proceeds more directly toward the desired equilibrium. The torque generated by $u_{P D}$ on the other hand is impeding the progress toward the desired equilibrium by reducing $|\dot{V}|$ from the value that would be achieved with zero control torque. 


\section{Conclusions}

The topological and geometric features of the global attitude stabilization problem have been discussed. The topology of the state space dictates that a globally asymptotically stabilizing control law must be discontinuous. Exponential coordinates, a minimal set of almost global coordinates, were used to represent the configuration space $S O(3)$. Jump conditions were specified along with the differential equations of motion to achieve a complete description of the state evolution. An appropriate attitude error was formulated and used for control design. Special issues in the Lyapunov stability proofs due to the topology of $S O(3)$ were identified and resolved. The simpler problem of stabilization for a fixed rotation axis, for which the configuration space is $S O(2)$, was also considered to provide insight.

Two new globally stabilizing attitude control laws have been derived using the inverse optimality method. These feedback control laws are optimal with respect to cost functions that penalize state errors and control effort, and yet they were obtained without solving the corresponding Hamilton-Jacobi-Bellman equations. Simulation results illustrated the performance advantages of these optimal stabilization laws over other globally stabilizing feedback laws that have appeared in the literature.

\section{Acknowledgments}

This research was supported by Rockwell and the California Space Institute. Helpful discussions with F. Landis Markley, John L. Junkins and Hanspeter Schaub during the course of this research are gratefully acknowledged.

\section{References}

1 Shuster, M. D., "A Survey of Attitude Representations," Journal of Astronautical Sciences, Vol. 41, No. 4, 1993, pp. 439-517. 
2 Meyer, G., "Design and Global Analysis of Spacecraft Attitude Control Systems," NASA Technical Report, NASA TR R-361, March 1971.

3 Crouch, P. E., "Spacecraft Attitude Control and Stabilization: Application of Geometric Control Theory to Rigid Body Models," IEEE Transactions on Automatic Control, Vol. 29, No. 4, 1984, pp. 321-331.

4 Ortega, R., and Spong, M.W., "Adaptive Motion control of Rigid Robots: A Tutorial",Automatica, Vol. 25, No. 6, 1989, pp. 877-888.

5 Koditschek, D. E., "The Application of Total Energy as a Lyapunov function for Mechanical Control Systems," Dynamics and Control of Multibody Systems : Proceedings of the AMS-IMS-SIAM Joint Summer Research Conference at Bowdin College, Maine, edited by Marsden, J. E., Krishnaprasad, P. S., and Simo J. C. , Vol. 97, American Mathematical Society, 1988, pp. $131-157$.

6 Wen, J.T.-Y. and Kreutz-Delgado, K., "The Attitude Control Problem," IEEE Transactions on Automatic Control, Vol. 36, No. 10, 1991, pp. 11481162.

7 Paielli, R. A., and Bach, R.E., " Attitude Control with Realization of Linear Error Dynamics," Journal of Guidance, Control and Dynamics, Vol. 16, No. 1, 1993, pp.182-189.

8 Bullo, F., and Murray, R. M., "Tracking for Fully Actuated Mechanical Systems: A Geometric Framework," California Institute of Technology - Control and Dynamical Systems CIT-CDS Technical Report, Feb. 1997.

9 Dwyer, T. A. W., "Exact Nonlinear Control of Spacecraft Slewing Maneuver With Internal Momentum Transfer," Journal of Guidance, Control and Dynamics, Vol.9, No. 2, 1986, pp. 240-247.

10 Wie, B., Weiss, H., and Arapostathis, A., "Quaternion Feedback Regulator for Spacecraft Eigenaxis Rotations," Journal of Guidance, Control and Dynamics, Vol. 12, No. 3, 1989, pp. 375-380.

11 Slotine, J.-E., and Di Benedetto, M.D., "Hamiltonian Adaptive Control of Spacecraft," IEEE Transactions on Automatic Control, Vol. 35, No. 7, 1990, pp. 848-852.

12 Egeland, O. and Godhavn, J.-M., "Passivity-based Adaptive Attitude Control of a Rigid Spacecraft," IEEE Transactions on Automatic Control, Vol. 39, No. 4, 1994, pp. 842-846.

13 Scrivener, S. L., and Thompson, R. C., "Survey of Time-optimal Attitude Maneuvers," Journal of Guidance, Control and Dynamics, Vol. 17, No. 2, 1994, pp. 225-233. 
14 Junkins, J. L., and Turner, J. D., Optimal Spacecraft Rotational Maneuvers, Elsevier, 1986.

15 Schaub, H., Robinett, R. D., and Junkins, J. L., "Globally Stable Feedback Laws for Near-minimum-fuel and Near-minimum-time Pointing Maneuvers for a Landmark-tracking Spacecraft," Journal of Astronautical Sciences, Vol. 44, No. 4, 1996, pp. 443-466.

16 Vadali, S. R., "Variable-structure Control of Spacecraft Large-angle Maneuvers," Journal of Guidance, Control and Dynamics, Vol. 9, No. 2, 1986, pp. 235-239.

17 Tsiotras, P., "Stabilization and Optimality Results for the Attitude Control Problem," Journal of Guidance, Control and Dynamics, Vol. 19, No. 4, 1996, pp. $772-779$.

${ }^{18}$ Freeman, R. A., and Kokotovic, P.V., "Optimal Nonlinear Controller for Feedback Linearizable Systems," Proceedings of the American Control Conference, Seattle, WA, June 1995, pp. 2722-2726.

19 Freeman, R. A., and Kokotovic, P.V., "Inverse Optimality in Robust Stabilization," SIAM Journal of Control and Optimization, Vol.34, No. 4, 1996, pp. 1365-1391.

20 Bharadwaj, S., Osipchuk, M., Mease, K. D., and Park, F. C., "A Geometric Approach to Global Attitude Stabilization," Proceedings of the AIAA/AAS Astrodynamics Conference, San Diego, August 1996, pp. 806-816.

${ }^{21}$ Osipchuk, M., Bharadwaj, S., and Mease, K. D., "Achieving Good Performance in Global Attitude Stabilization," Proceedings of American Control Conference, Albuquerque, New Mexico, June 1997, pp. 1889-1893.

${ }^{22}$ Krstic, M. and Tsiotras, P., "Inverse Optimality Results for the Attitude Motion of A Rigid Spacecraft," Proceedings of the American Control Conference, Albuquerque, NM, June 1997, pp. 1884-1888.

${ }^{23}$ Chevalley, C., Theory of Lie Groups, Princeton University Press, Princeton, 1946.

${ }^{24}$ Park, F. C., The Optimal Kinematic Design of Mechanisms, Ph.D. thesis, Harvard University 1991.

25 Bhatia, N.P., and Sezgö, G. P., Dynamical Systems : Stability Theory and Applications, Springer-Verlag, 1967.

${ }^{26}$ Boothby, W. M., An Introduction to Differentiable Manifolds and Riemannian Geometry, 2nd Edition, Academic Press, Orlando, 1986, pp. 187-192. 
27 Bullo, F., and Murray, R. M., "Proportional Derivative Control on the Euclidean Group," Proceedings of the Third European Control Conference, Rome, Italy, Sept. 1995, pp. 1091-1097.

${ }^{28}$ Schaub, H., Junkins, J. L., and Robinnett, R. D., "New Penalty Functions and Optimal Control Formulation for Spacecraft Attitude Control Problems," Journal of Guidance, Control and Dynamics, Vol. 20, No. 3, 1997, pp. 428434.

${ }^{29}$ Isidori, A., Nonlinear Control Systems, 2nd Edition, Springer, New York, 1995, pp. 283-288.

${ }^{30}$ Colbaugh, R., Barany, E., and Glass, K., "Global Stabilization of Uncertain Manipulators Using Bounded Controls", Proceedings of the American Control Conference, Albuquerque, New Mexico, June 1997, pp. 86-91.

${ }^{31}$ Mortensen, R. E., "A Globally Stable Linear Attitude Regulator", International Journal of Control, Vol. 8, No. 3, 1968, pp. 297-302.

32 Bilimoria, K. D. and Wie, B., "Time-optimal Three-axis Reorientation of a Rigid Spacecraft," Journal of Guidance, Control and Dynamics, Vol. 16, No. 3, 1993, pp. 446-452.

${ }^{33}$ Freeman, R.A. and Primbs, J.A., "Control Lyapunov functions: New Ideas from an Old Source", Proceedings of the 35th Conference on Decision and Control, Kobe, Japan, December 1996, pp. 3926-3931. 


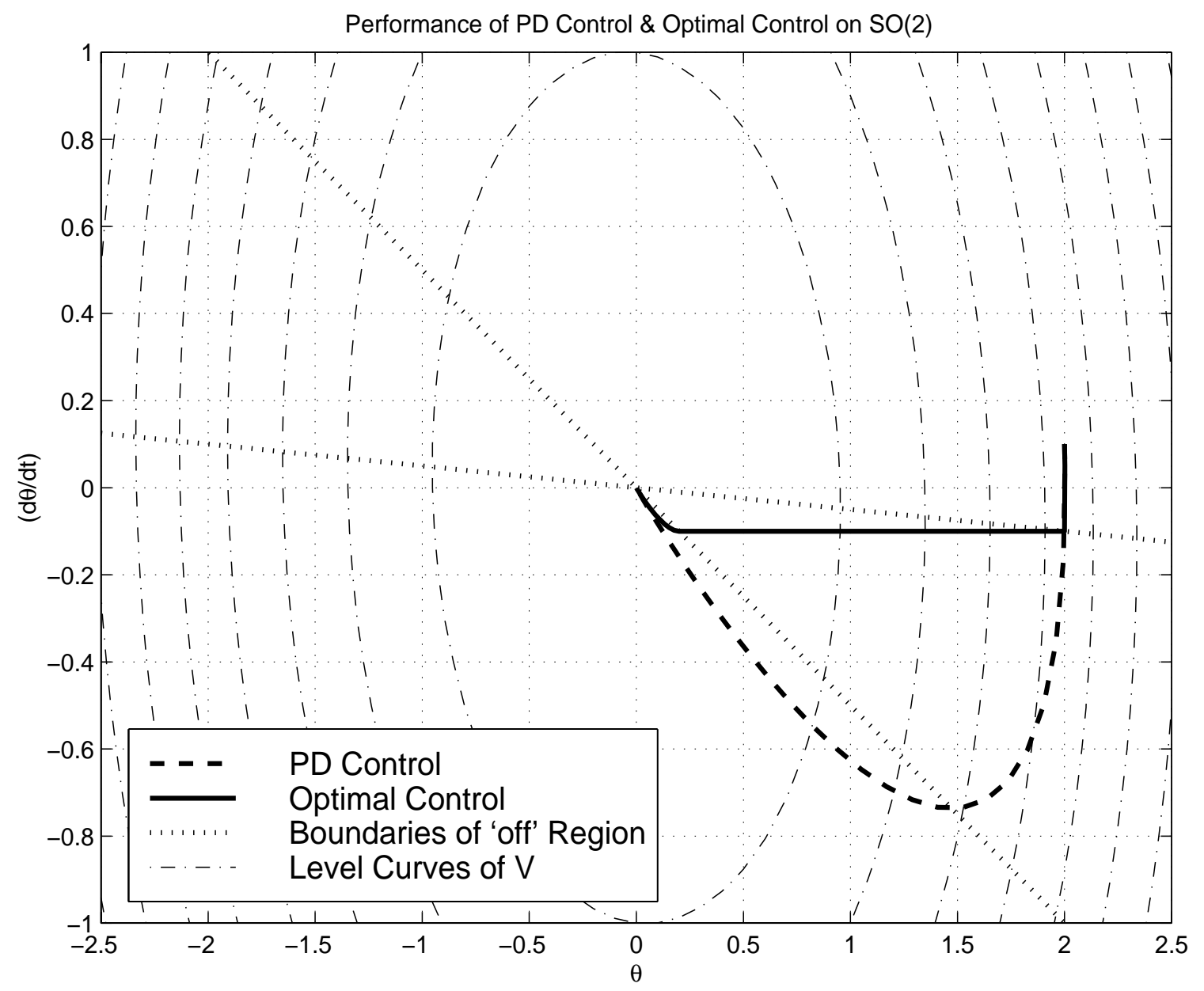

Figure 1: Comparison of PD control and optimal control on $S O(2)$. 


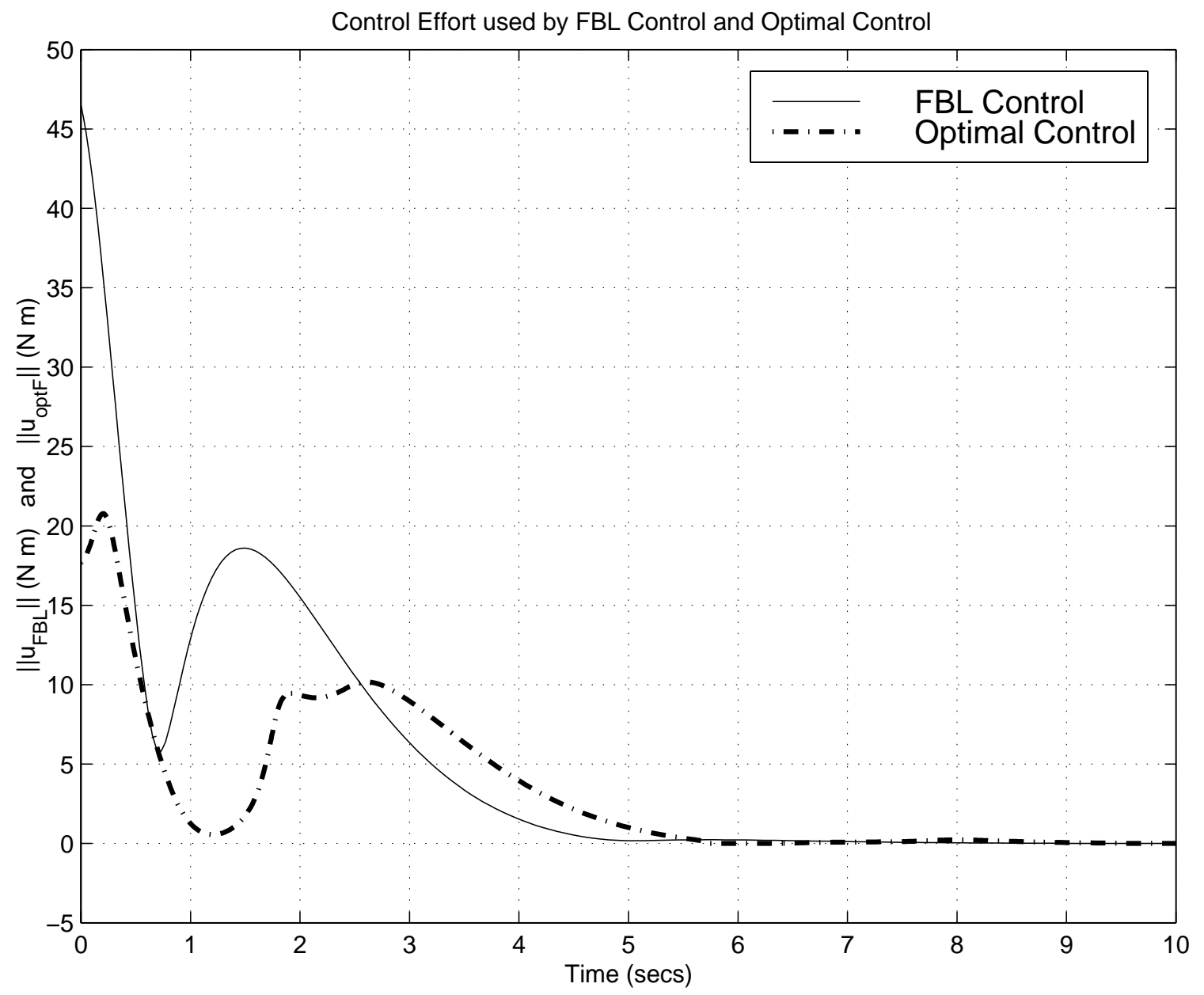

Figure 2: (a) Comparison of $\left\|u_{F B L}\right\|$ and $\left\|u_{o p t F}\right\|$ vs. time. 


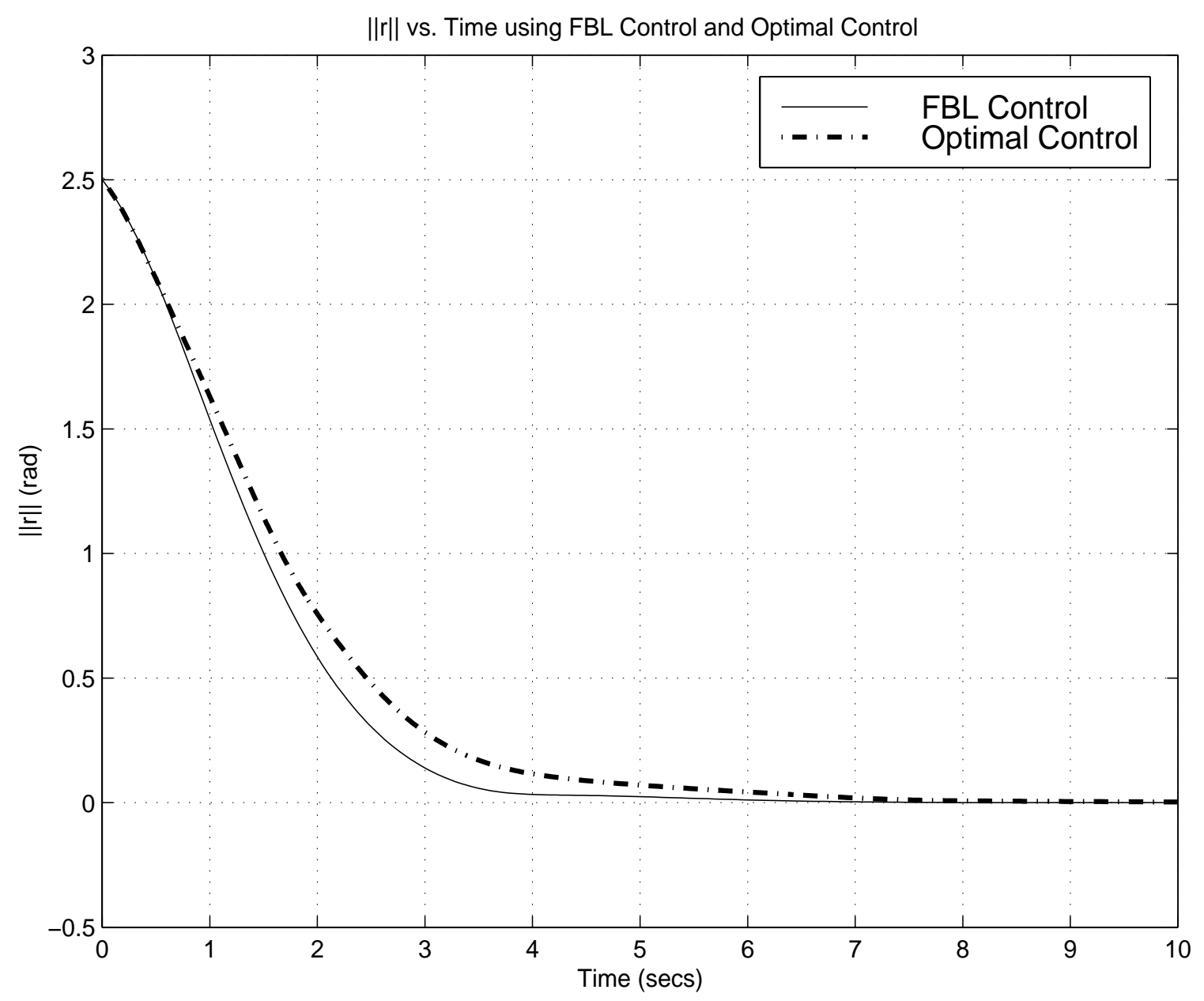

Figure 2: (b) Comparison of $\|r\|$ using $u_{F B L}$ and $u_{o p t F}$ vs. time. 


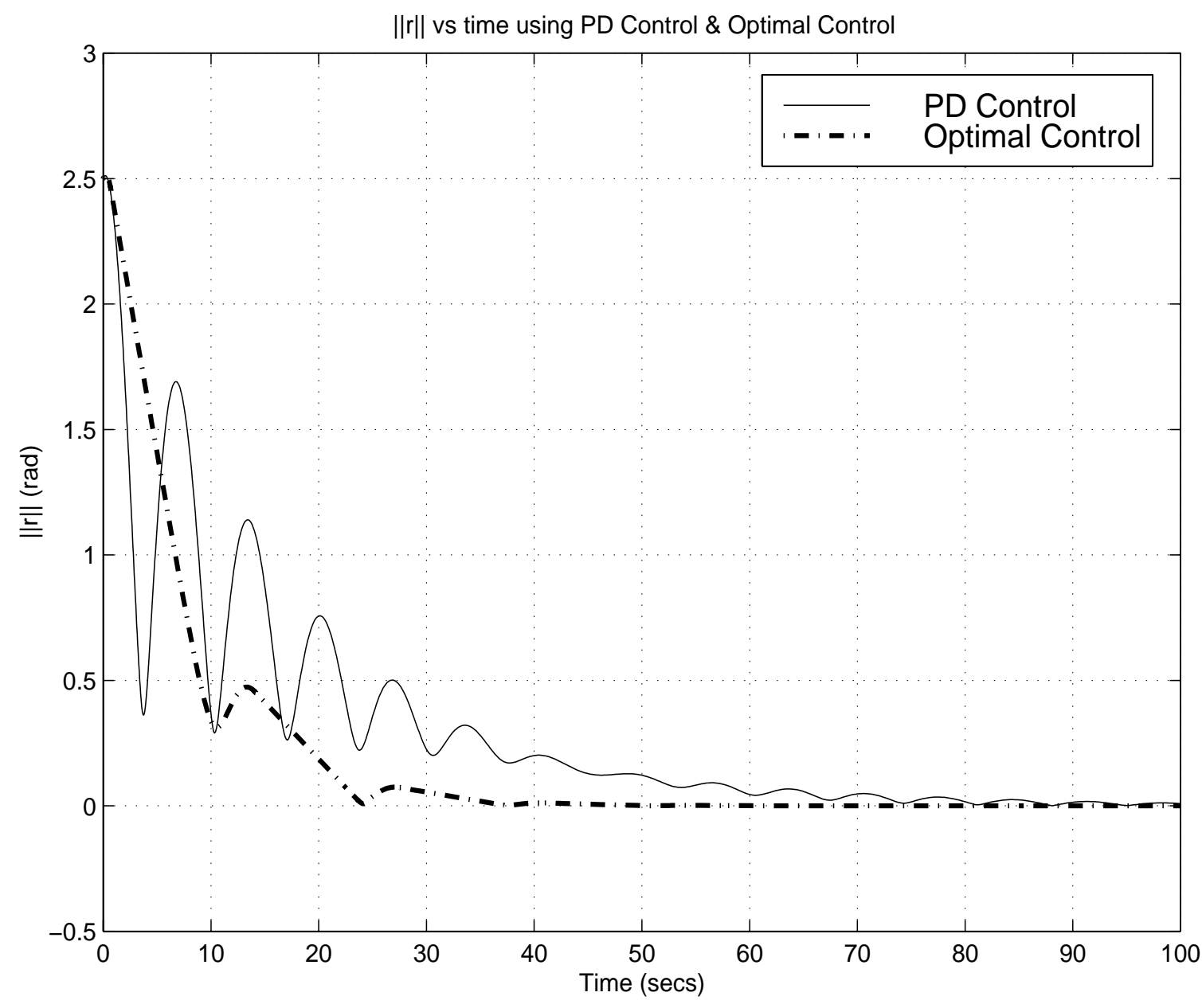

Figure 3: (a) Comparison of $\|r\|$ using $u_{P D}$ and $u_{o p t E}$ vs. time. 


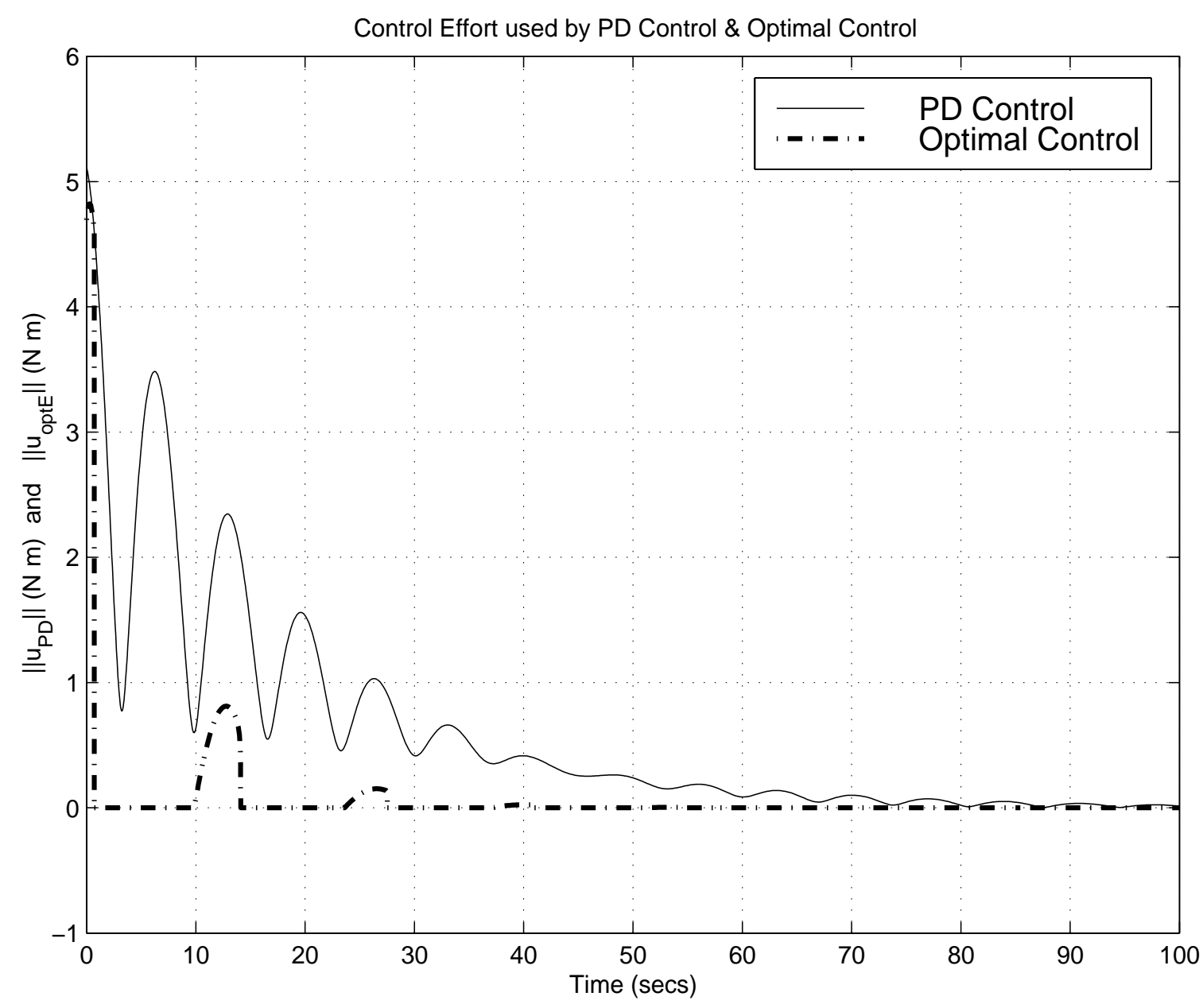

Figure 3: (b) Comparison of $\left\|u_{P D}\right\|$ and $\left\|u_{o p t E}\right\|$ vs. time. 


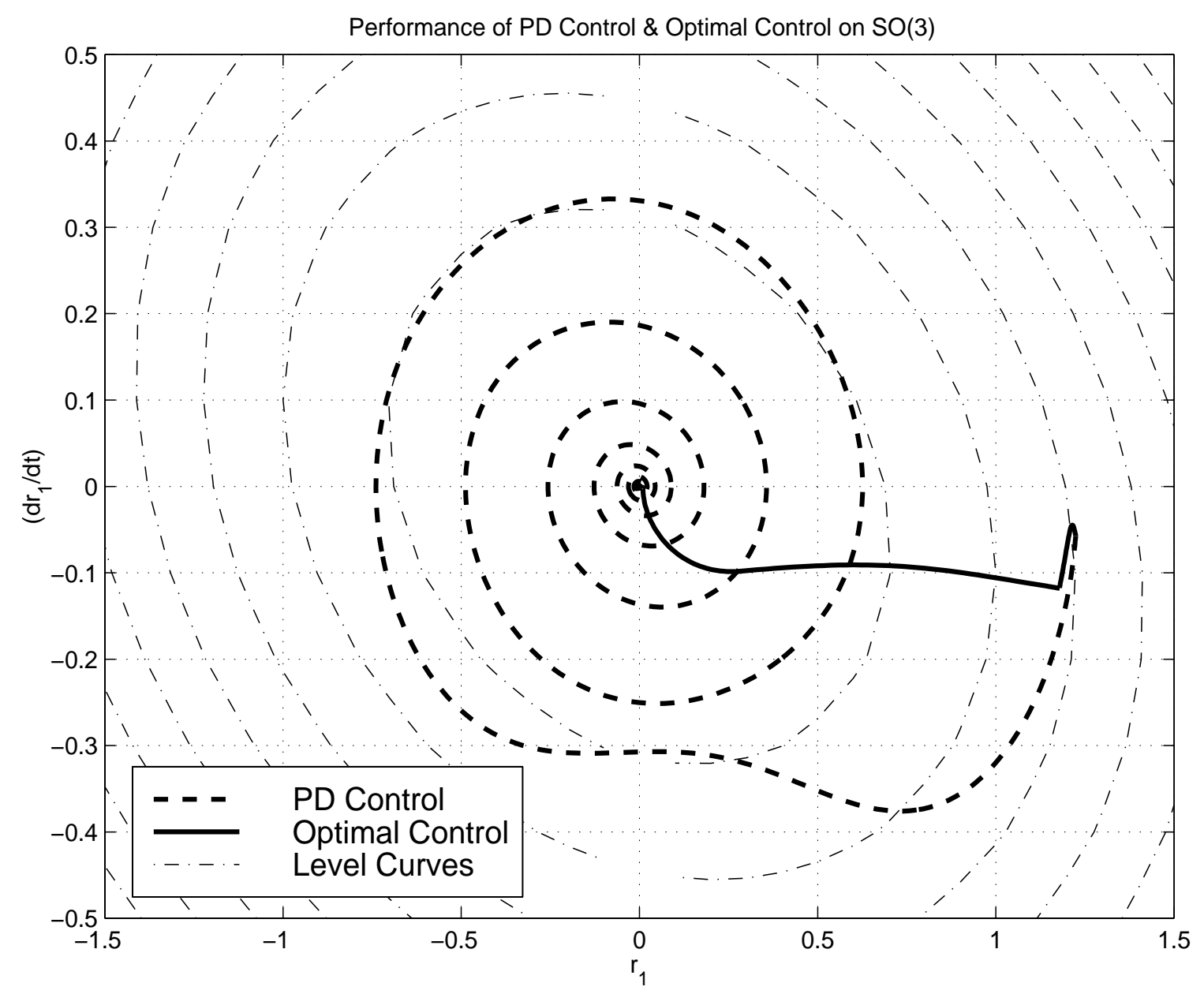

Figure 4: Comparison of trajectories using $u_{P D}$ and $u_{o p t E}$ on a slice of the state space. 
Fig 1. Comparison of PD control and optimal control on $S O(2)$.

Fig 2(a). Comparison of $\left\|u_{F B L}\right\|$ and $\left\|u_{o p t F}\right\|$ vs. time.

Fig 2(b). Comparison of $\|r\|$ using $u_{F B L}$ and $u_{o p t F}$ vs. time.

Fig 3(a). Comparison of $\|r\|$ using $u_{P D}$ and $u_{o p t E}$ vs. time.

Fig 3(b). Comparison of $\left\|u_{P D}\right\|$ and $\left\|u_{o p t E}\right\|$ vs. time.

Fig 4. Comparison of trajectories using $u_{P D}$ and $u_{o p t E}$ on a slice of the state space. 